\title{
Exploring the characteristics of active health seekers, the thinking behind patient preferences, and the implications for patient-professional relationships

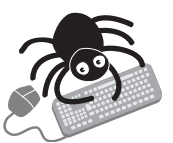

Please visit the QSHC website (http://www.qshc.com) for links to these articles - many to full text.

The papers summarised below will provoke reflection on the likely effects of empowered and empowering patients upon the professional-patient relationship.

Exploring "healthism" in the middle classes - In the first paper a GP and psychiatrist explore what many health professionals see as a common, increasingly uncontainable, and personally stressful problem: the beliefs, behaviour and expectations of the articulate, health aware, and information rich middle classes. The issue is encapsulated in the phrase "healthism" which "is related to, but should not be equated with, consumerism, which is a broader term with a host of different meanings, depending on context ${ }^{\prime \prime}$.

Consumerism has been described as having five faces: a vehicle for power, the ideology of conspicuous consumption, an economic ideology, a political ideology, and a social movement to protect the rights of consumers. "This last meaning comes closest to the positive connotations of consumerism as applied in health care. But the more negative notion .... is probably the meaning that aligns best with healthism as an individual behaviour pattern and a potential public health problem." The paper describes some fictitious case scenarios to illustrate "healthism" and its effects. They reflect real life problems encountered in clinical situations. For example:

Case 1: Fiona and Bruce Taylor-Brown are a childless couple in their mid 30s. Both work full time in the financial sector and have difficulty attending normal surgery hours. They are not frequent attenders, but when they do come, they always book the last appointment of the evening and usually arrive late, just as the GP is locking up. One Friday evening, Bruce attends complaining of a 2 week history of generalised weakness, light headedness, and "a burning feeling going around in the blood". He has with him a printout from the Internet which attributes his symptoms to the presence of mercury in dental amalgam. He also has the business card of a private consultant who will remove his amalgam fillings on referral from his GP. He wants the GP to sign a claim form so that he can have this 'emergency' treatment reimbursed. The doctor is not happy about making a referral for what she sees as an unnecessary referral and refuses to sign. At this point, the receptionist phones through to announce that [Bruce's partner] Fiona has arrived and is knocking on the window demanding to be let in, even though it is now 40 minutes past the official closing time.

Case 2: River is a healthy breastfed baby born at term in a birthing pool. At the baby's 6 week check the health visitor advises that River should receive the standard programme of immunisations. At this point the child's mother becomes upset and begins to murmur about one-sided paternalistic advice. She brings out a press cutting that exposes GPs financial motives for promoting "unsafe" vaccines.

Case 3: the third case is a divorced housewife in her mid 50s. Ten years ago she had breast augmentation and now is complaining of aching in the neck and shoulders and difficulty sleeping. Physical examination by the GP confirms some tension of the trapezius muscle and mild restriction of the expected range of movement of both shoulder joints. "I knew it", she exclaims. "I've got fibromyalgia from the silicone breast implants". She asks the GP to write a letter confirming the diagnosis so she can approach a legal firm specialising in compensation claims,

Despite "the wealth of publications on the impact of healthism, there is surprisingly little published research available on the nature of the sociocultural phenomenon". The authors suggest a number of drivers:
- Technological advances resulting in reduced mortality from acute infectious diseases.

- A conflation of health not just with the absence of disease but with total physical, social, and psychological well being,

- Declining fertility rates alongside (for some) rising leisure time and disposable income.

- Rise of the consumerist movement.

- General trends in western society towards personal reflection and self-awareness.

- Widespread commercialisation of health.

- Progressive medicalisation of all aspects of daily life including food choices, leisure activities, mood changes and coping with life events.

The authors see "healthism" as an "ideologically insidious force" which elevates health to "a metaphor for all that is good in life". They say the pharmaceutical industry has contributed to this process by "extending the boundaries of diagnostic entities such as social phobia, attention deficit hyperactivity disorder and obsessive compulsive disorders as part of a conscious campaign to increase consumer awareness and hence expand markets".

While healthism is presented as a middle class phenomenon and, of course, consumerism in general depends on disposable income, the authors suggest that although the behaviours and attitudes are strongly class related, the symptoms are not. For example, research examining chronic fatigue syndrome-popularly characterised as "yuppie flu"-have found it is actually commoner in lower socioeconomic groups.

The question is, then, why have these conditions become seen as middle class complaints? The authors argue that, while all groups are likely to complain of symptoms, the middle classes are more likely to receive specialist treatment "presumably because they [are] better equipped to persuade GPs to refer". They conclude that "whilst many of the drivers behind our modern culture of health and well-being are beyond the scope of medicine, health professionals need to be aware of the healthism phenomenon if they are to understand patient concerns, avoid medical encounters [that are] unsatisfactory for both parties, and make the best use of limited resources"

A Greenhalgh T, Wessely S. "Health for me": a sociocultural analysis of healthism in the middle classes. Br Med Bull 2004;69:197-213.

What kind of people surf the Internet for information? - An increasing number of individuals are using the Internet to meet their health information needs; however, little is known about the characteristics of online health information seekers and whether they differ from offline sources. Cotton and Gupta argue that "a more complete understanding is required of the extent to which online health information is penetrating people's lives, the scope of the impact it will have on them, and implications of seeking online health information". "Above all", they say, "we must discover the primary characteristics of online and offline health information seekers to better recognize their needs, highlight improvements that may be made in the arena of Internet health information quality and availability, and understand factors that discriminate between those who seek online vs. offline health information".

The authors find it particularly interesting that, despite the enormous volume of information searches, relatively speaking, few patients are likely to display information seeking behaviour in the company of their doctors. "This may be a function of either the hierarchical relationship between patients and providers and/or the declining length of appointment times". Yet the balance of power/ nature of consultation may change "as health management 
organisations become less lenient and the burden of responsibility for patient knowledge and choices fall increasingly on patients".

There are plenty of hypotheses about preferences for Internet searching. One the researchers cite is that it "affords individuals privacy, immediacy, convenience, anonymity, a wide variety of information, and a variety of perspectives on the same topic". An interesting finding is that users when searching tend not to identify themselves. One study found that only $21 \%$ of health seekers have disclosed their email address when visiting a health related website and only $17 \%$ provided any personal information.

To explore the characteristics of health surfers in more detail the researchers drew on a sample of the 285 people who responded to the 2000 General Social Survey. The subsample is not typical of the whole General Social Survey sample: it is slightly more likely to be more female and non-married and likely to have higher levels of education. Those who search for health information online are younger (mean age $40 \vee 52$ years), have higher incomes and are better educated $(48 \%$ of the Internet health seekers report having a degree compared with $18 \%$ of the whole sample). Online health information seekers also report better well being when assessed through self-reported health status. $86 \%$ of the online group reported good health status compared with $60 \%$ of the offline group.

The two groups are very different and, crucially, for the researchers, "similar to those reflected in the digital divide literature". "Examination of the total canonical structure coefficients reveals that income, education, age and health are the dominant variables. Their signs suggest that we might refer to the discriminant function as a general inequality/digital divide index".

Given that the online seekers tend to be healthier and more proactive in managing their own health, an important implication of the research is that governments and health services should seek to find ways of increasing Internet access, training and comfort of use. But there is a broader point. "Aspects related to larger structural inequalities in US society appear to play key roles in discriminating between online and offline health information seeking. A policy implication of these findings is that diminishing these inequalities may be the key to increasing online health information seeking and thus potentially empowering health care consumers."

- Cotton S, Gupta S. Characteristics of online and offline health information seeks and factors that discriminate between them. Soc Sci Med 2004;59:1795-806

The quest for cancer information on the Internet - Research and "optimistic commentators" argue that wider access to medical information will encourage "a more balanced encounter between patient and health professional" , that it will lead to better informed decisions, a stronger relationship and greater satisfaction all round. Yet, not all health professionals have been enthusiastic about the increase in public access to health information. One of the arguments used to support this relates to equity. "It has been suggested that an inverse information law operates whereby those who are in the greatest need of information about preventable or treatable conditions are least likely to have access to new technologies". Furthermore, it is argued that it is unreasonable to expect patients to be able to evaluate the vast quantities of health information they find.

This paper draws upon in-depth interviews with British men and women with cancer $(n=175)$ but focuses on two in particular, a woman with inflammatory breast cancer and a man with prostate cancer. It aims to demonstrate how the Internet has been used not only to gather information and gain support from others, but also how it helps people make sense of the experience of cancer. Although the paper only focuses on two cases and it is not claimed they are statistically representative, it is suggested that they "symbolise, portray and represent something important about the experience of illness".

Case 1: Patricia is a teacher in her mid 40s "who experiences a sudden pain in her breast one Sunday evening". Her initial encounter with the health service was not positive. Her doctor would not see her and asked her to see the practice nurse instead. The nurse dismissed the lump as "hormonal and told her not to worry"; she was advised to "go away and keep a diary". Patricia felt that she "wasn't worthy" of being seen by a doctor and was frustrated the nurse did not examine her. She was frightened. Over the next two days the pain became worse and she returned to the practice. Despite the nurse having initially told Patricia she was not qualified to examine breasts, this time she did, in the presence of the doctor. After the examination she was sent straight to hospital. The cancer was confirmed, she had chemotherapy, a mastectomy, and then radiotherapy. At the time Patricia was too focused on getting through the treatment to seek much information, "but once the radiotherapy finished she found she wanted to know more about IBC ... So she started to research on the Internet". She wanted to make sure she had a good prognosis. She joined an Internet support group for women with IBC, which has around 600 members and provides a sense of community and expertise that would be hard to find in a local UK support group. The group was particular helpful when she was unsure how to talk to her 11 year old son about her illness. Patricia moved from a quest for information to become a campaigner for it. She has become aware that IBC is a quite rare condition, and that there is a danger that the symptoms could be confused with mastitis, causing what may be dangerous delays since the condition develops fast. She has therefore embarked on a mission to ensure that women know about it and that GPs know when a woman should be referred. She says she has moved from away from being the sort of person who would run from "anything that even smelled of me having to fight" to a position where "I'm like a bull dog".

Case 2: Graham, a self-employed engineer, discovered he had prostate cancer as an indirect result of an emergency admission. Initially he was given no information. "I sat down that very evening at the computer and started to do the stacking up and learning about it ${ }^{\prime \prime}$. He constructed a table on his computer to guide his choice, in which he noted the pros and cons of the different treatments. Impotence and incontinence are two of the most common side effects of treatment, but Graham considers that many men are too bothered about potency: for him the "real question" was "what is the best treatment to keep me alive?" The researchers point to two aspects of Graham's story: "his moral evaluation of the behaviour of his doctors" and "the importance of his professional identity as an engineer". Each contributed to his "quest" for information. When he first began to feel ill his GP refused to make a home visit, "even though Graham was coughing up blood". So he found out when the shifts changed and telephoned again when a different doctor was on duty. The second doctor admitted him to hospital immediately and the cancer was discovered. He remains critical of his GP for refusing to discuss or accept the research he uncovered about how to manage his disease and contrasts this with his urologist who, though at first was reluctant, has over time followed up references. "He's the only one I can say who's done that, all the rest have said: 'Hey, you think you want to be a doctor. I'm not treating you'.

"In Graham's account the need to discover information about treatment for himself is important because without it he would have received a treatment that he saw as less than optimum" (radical prostatectomy). His professional identity, he says, provided him with problem solving skills, Internet savvy and a confidence to question the evidence for the standard treatment. He clearly believes his own initiative helped him survive. "Graham states at several points in the interview that he had no assistance whatsoever from the UK medical profession. He says that neither his GP nor his consultant urologist provided information about different treatment choices. He mentions that he has exchanged atrocity stories about misdiagnoses and mismanagement of prostate cancer via a prostate cancer support group and correspondence with American Internet sites."

The authors say the interviews in this paper were chosen because they raise important issues about the way that the Internet is changing how people with cancer experience their illness and engage with health professionals. The researchers acknowledge that relying solely on the stories of patients does not give a full insight into patient-professional relationships in this context, and call for further research to understand how the Internet is changing dynamics.

A Ziebland S. The importance of being expert: the quest for cancer information on the Internet. Soc Sci Med 2004;59:1783-93

Reasons underpinning patient preferences for angina treatments The authors of a paper in Health Expectations begin by noting that their "systematic review of the literature on patients' perspective on patients' preferences in clinical decision making reported relatively little literature on the patients' perspective, and focused mainly on the challenges for doctors in involving patients in decisions". Finding ways to elicit patient preferences is not easy, yet they are critical "in building up a patient based ethics of evidence when there is uncertainty about when to 
provide more or less intensive or invasive treatments to patients, and when issues of health service prioritisation are being debated".

The sample for the paper were recruited from two general practices in Norwich, They wanted patients who had been diagnosed with angina within the past 5 years. Once patients had responded to a letter of invitation from the GPs, they were telephoned by a researcher to arrange an interview at their surgery. Of the 21 people interviewed, 13 were male and eight female. The youngest was 59 and the oldest 80 . The mean age was 68 . The vast majority left school between 14 and 16 and none had received any form of higher education.

In interviews, patients were shown seven descriptions of potential treatment options. The treatment options presented on laminated cards were: (1) no treatment, (2) drug therapy to treat symptoms only, (3) drug therapy to prevent occurrence of chest pain/ breathlessness, (4) drug therapy to prevent symptoms occurrence and reduce risk of future heart attack, (5) balloon angioplasty, (6) coronary artery bypass surgery, (7) keyhole surgery. The task was introduced by the interviewer as follows: "Imagine that your angina symptoms were slightly worse than they are now and you went to your GP, who offered you three and only three possible treatment options". Individuals were asked to indicate their most and least preferred item and the reason for their choice. All felt the "no treatment" option was worthless. Most preferred option 4 (drug therapy to reduce future symptoms). Yet they were probably biased to this view as it is the form of treatment most are receiving. Generally, all three of the drug therapy options were preferred to surgical interventions.

The most interesting aspect of the paper is its exploration of the reasons given for their treatment preferences. Analysis elicited 27 reasons behind the rankings given to treatments. The most mentioned (by 21 out of 21) was "worthless" - in relation to the no treatment option. The second most cited reason is the opposite"effective" - which was used in relation to drug therapy and surgical options and mentioned by 18 of the 21 . However, "it was clear that for some patients, there was no one type of treatment that was always most effective, and that effectiveness was seen as contingent upon the severity of symptoms". The third most cited reason is labelled "appropriate for severe symptoms", used by 17 of the 21. Most of the participants could not ("or did not want to") imagine their symptoms worsening, but they could countenance an invasive treatment as a last resort. 16 of the 21 talked about "risk" as a form of reasoning used exclusively in relation to surgical and invasive treatment options. 13 of the 21 used a closely related reason - "bad effects from procedure" - to discuss side effects. This applied to all treatments. One said they tried not to use nitrite spray. "I don't like it and gear any activity, even walking the dog or anything like that, you know, the pace, so I don't have to use that. As soon as I feel the tightness start to come I slow down". For many of the patients the mere thought of surgical procedures and their frightening nature drove choices. "Upon probing, this reason seemed to reflect a strong reluctance to be physically cut or have their bodies interfered with".

Personal knowledge and the relaying of others' experience is a critical factor in making choices and can play positive or negative roles. Someone who had successful surgery or drug treatment could be a guiding influence. Bad experiences put people off treatments. "We've lost a cousin with that one (CABG), so that puts me off straight away. He came home alright ... but within six months he passed away" (collapsed with a heart attack).

The reasons above were important to almost half the group. But other reasons are worth noting because they reflect more personal preferences. They include "suitable for maintaining current lifestyle" $(\mathrm{n}=8)$, "suitable for old people" $(\mathrm{n}=5)$, "expensive for NHS" $(n=2)$, "likely to result in burden on carers" $(n=2)$, "too much time in hospital" $(n=1)$, "dependent on skills of others" $(n=1)$.

There may be a generational explanation, but among this older group of people many would prefer their doctor to make the decision.

"I wouldn't want to just take their work ... I'd want to know why, but I think it's probably unlikely that I would go against [the doctor's advice $]^{\prime \prime}$.

"I don't see the point in going to the doctor if you don't take any notice of what they say".

The cards shown to patients enabled them to think through options and helped them decide. The author suggests that this is "an area where better information for patients, and access to patient support groups, on the effectiveness and timeliness of interventions, as well as clearly presented, simple information about risk is needed". "The use of laminated show cards was not intended for use as a clinical tool, but the issue of whether doctors could use them with patients to facilitate any joint decision-making process is a question for further research".

$\Delta$ Lambert $\mathrm{N}$, et al. Reasons underpinning patients' preferences for various angina treatments. Health Expectations 2004;6:246-56.

Examining follow up choices after treatment for colorectal cancer "The rationale behind clinical follow up after cancer treatment is that recurrent disease will be detected early, thus reducing morbidity and mortality". In this paper, the authors set out to describe current hospital follow up policy and to explore patients' needs and preferences for follow up.

A survey was undertaken of 50 hospitals that were taking part in the colorectal cancer collaborative. Interviews with 20 men and 19 women were also undertaken during 2001/2002. The researchers recruited volunteers by giving a recruitment pack to GPs, consultants, nurse specialists and support groups and people who wished to volunteer returned a contact slip direct to the researcher. Because of this approach it was not possible for the research team to calculate a response rate. Hospital follow up policies at this time were not extensive. Only three off the 35 hospitals specifically stated that patients were given a choice about the type of follow up. Yet, in interviews, patients' needs for support and information and their different attitudes to follow up care were strong emergent themes from the qualitative thematic analysis. The patients' accounts highlight the importance of tailoring follow up care to individual preferences.

"Many people reported feeling cut adrift once they were discharged from hospital and pointed out that their recovery would have been less traumatic if they had been given a more realistic idea of what to expect". They described needs for realistic information about recovery, a responsive GP, non-contradictory advice about diet, and information about resources. "The need for realistic information about the possibility of adhesions was underlined by the experience of some people who were not warned and were extremely distressed when flare-ups occurred".

One of the problems, chiming with the experience of Graham reported in the paper above, was that difficulty in diagnosing the cancer and delays in referral had "seriously undermined some people's relationship with their $\mathrm{GP}^{\prime \prime}$. This made it difficult to engage them in follow up care.

Sometimes GPs did not know what follow up involved. "He said he'd had a letter from the doctor at the hospital to say he wasn't seeing me anymore unless it was necessary. And I said, 'Well, I understand that I will have to have an ultrasound scan' which has come along since, because I recently saw the surgeon and he um, he advised, or he said, 'when did you have your last scan?' I said, 'I had one before the operation. So he said, 'Well, I think its time you had one of those then ...' And my GP said to me, 'Well you probably know more than I do'. He said, 'So, yes, I suppose it's normal to have these follow up treatments' he said 'but I don't really know'. So I don't think the GPs are aware."

The importance of being given the telephone number of a specialist nurse was mentioned by several patients as helping to boost their confidence during the early stages after treatment. Such information was rarely given. Diet was a particular difficulty, knowing what and what not to eat.

The importance of involving individual patients with colorectal cancer in choices about the nature and setting of their follow-up is apparent. While some respondents valued the reassurance of visiting a hospital for follow up scans and colonoscopy, the anxiety of contemplating the visit and waiting for test results contributed to less positive views. The interviews led the researchers to suggest "that patients may choose less costly options, allaying concerns that involving patients might increase costs without tangible benefit".

The authors suggest a checklist of issues for GPs to discuss at a colorectal follow up appointment:

- discussion of the reasons for any delay in diagnosis;

- expression of willingness to help to seek any information the patient needs;

- details of sources for information; 
- dietary advice;

- an explanation of what to expect during recovery, possible complications and what to do if concerned;

- details of support groups;

- discussion of options and preferences for follow up care.

$\Delta$ Rozmovits L, Rose P, Ziebland S. In the absence of evidence, who chooses? A qualitative study of patients' needs after treatment for colorectal cancer. J Health Serv Res Policy 2004;9:159-64.

What factors influence patients' judgements of medical errors? "Medical errors are among the most serious quality problems in health care systems and are associated with considerable health related harm and economic burden". They also have the potential to compromise the patient-doctor relationship and to undermine patients' trust in the system. This paper explores how doctors' handling of errors affects patients' evaluations of the incident and views on what action should be taken.

A German survey panel was presented with a series of hypothetical scenarios involving a medical error. Each vignette was followed by the same four questions: (i) rate the severity of the incident, (ii) whether participants would seek referral to another physician, (iii) whether the incident should be reported to higher authorities, and (iv) the kinds of consequences the physician should face. Each respondent $(n=984)$ provided some demographic data and attention was paid to age, sex, education, income, occupation, health cover, and the extent to which they use the Internet. Two other questions were asked: whether respondents had experienced a medical error and if they were fearful of errors when receiving health care.

Thirty percent of respondents reported that they had experienced an error in their own care. Nearly $70 \%$ provided extensive descriptions of their experiences indicating that the majority did indeed have specific events in mind when answering. The judgements of error varied. While gender and income were not found to be significant in determining severity judgements, education was. Higher educated responders rated the described error as less severe and were less likely to pursue action. Unexpectedly, having experienced an error in one's own care did not affect respondents' appraisal. However, being afraid of errors did contribute to the probability of negative judgements.

What is really interesting in this paper is that the crucial variable in judging severity and prescribing action is "the physician's approach to the error". Extensive feedback was provided with the survey and it tended either to praise honest acknowledgement and courage or to criticise disrespect and self-righteousness. The researchers computed best/worse case scenarios for each model, comparing the probability of negative judgements following the best versus the worst possible approach to the error. The only differences between the scenarios was the communication style (comprehensive versus narrow), the acknowledgement of error as such, and whether the physician apologises.

The research provides evidence that "full disclosure of errors may strengthen rather than undermine the relationship between patients and physicians". This is not to say that professionals can get away with half-hearted acknowledgements because participants in the study were "very sensitive to precisely such attempts to mislead patients and did not accept professionals muddling through the issue elegantly".

The authors conclude that it is the revealing of deception that erodes patients' trust and this "sense of betrayal" outweighs any distress from being told the truth. The relabelling of errors as, for example, unhappy events should be strongly avoided since it erodes patients' trust and increases distress.

A Schwappach D, Koeck C. What makes an error unacceptable? A factorial survey on the disclosure of medical errors. Int J Qual Health Care 2004; 16:31726

T Smith

Senior Policy Analyst, Health Policy and Economic Research Unit, British Medical Association, London WC1H 9JR, UK; 